\title{
Integral Based Approach for Determining Motion Vector Fields
}

\author{
Atsushi Nomura \\ Department of Cultural and International Studies, Yamaguchi Prefectural University \\ Sakurabatake 3-2-1, Yamaguchi, 753 Japan
}

\begin{abstract}
This paper describes a method determining a motion vector field from an image sequence. In this method, a couple of a basic constraint equations in integral form and an additional constraint equation of spatio-temporal local constancy of a motion vector field is solved by the over-deterministic approach such as the least squares method. The method is applied to artificially synthesized image sequences and a real image one. As the results, robustness of the proposed method to noisy image sequences is confirmed. In addition, it is confirmed that the method requires less calculation cost than a conventional spatio-temporal optimization method.
\end{abstract}

\section{Introduction}

A motion vector field represents an apparent velocity vector field of brightness patterns in an image sequence. When a TV camera is translated in a static real world, we can obtain 3-dimensional structure of the world from the motion vector field [1]. Since recovering the 3 -dimensional structure is useful for object recognition, the reliable method determining the motion vector field is highly required in computer vision research. In the research field of scientific measurements, visualization techniques of scientific phenomena have been developed recently. For example, fluid flow can be observed by tracer particles and a slit light laser illumination system. Besides this, dynamics of a human heart is visualized by magnetic resonance imaging (MRI). Thus, determining velocity vector fields from image sequences is commonly required for understanding the phenomena.

Many methods determining the motion vector fields have been proposed. Most of the methods are divided into the matching based approach and the gradient based one. The matching based approach traces a brightness pattern or a feature point (spot) between two successive images by using a mutual correlation function [2]. Then the displacement vector field is obtained. On the other hand, the gradient based approach utilizes a basic constraint equation relating brightness distribution of an image sequence $f(x, y, t)$ to two motion vector components $\left(v_{x}, v_{y}\right)$. A typical basic constraint equation [3] is,

$$
\frac{\partial f}{\partial t}+v_{x} \frac{\partial f}{\partial x}+v_{y} \frac{\partial f}{\partial y}=0 .
$$


A motion vector is determined by solving a set of the basic constraint equation and additional constraints representing nature of the vector field, such as smoothness [3], spatial constancy [4] and temporal stationariness [5]. In consequence, appropriate constraint equations should be applied to an image sequence for the recovery of reliable motion vector fields. For instance, if an image sequence has much noise in brightness level, constraint equations having the effect of reducing the noise should be applied to that.

In addition to the above constraint equations, some researchers proposed new basic and additional constraint equations. For example, Aisbett proposed the extended basic constraint equation including a divergence term of a motion vector field and a rate of brightness generation which represents the brightness change of a pattern under illumination change [6]. Bimbo et al. analyzed the divergence term and confirmed its usefulness [7]. Some researchers proposed additional constraint equations on the rate of brightness generation $[8,9]$.

In this study, a basic constraint equation in integral form is proposed for the determination of an accurate motion vector field. In particular, the determination of the motion vector field from a noisy image sequence is focused on. Furthermore, the basic constraint equation is coupled with the simple additional constraints which have been already proposed [10]. The set of the equations are solved by the least squares method. The proposed method is applied to artificially synthesized image sequences and a real image one. Through the analysis of the image sequences, the usefulness of the proposed basic constraint equation is confirmed.

\section{Theory}

The conventional gradient based approach utilizes the differential form of basic constraint equations, say eq.(1). Since the equations have differential operations: $\partial f / \partial x, \partial f / \partial y, \partial f / \partial t$, they are not so robust to noise. In contrast to this, integral operations are expected to be robust to noise. Therefore, in this paper, a basic constraint equation having integral operations is introduced,

$$
\frac{\mathrm{d}}{\mathrm{d} t} \int_{\delta S} f \mathrm{~d} s=-\oint_{\delta C} f v \cdot n \mathrm{~d} c
$$

where $\delta S$ is a spatial domain surrounded by $\delta C, v$ is a motion vector to be determined and $\boldsymbol{n}$ is a unit length vector normal to $\delta C$ and pointing to its outside (Fig.1). The equation shows that the total brightness change observed in $\delta S$ is represented by movement of brightness pattern into $\delta S$. (This is known as Euler's observation method in fluid physics.)

Now we propose a new method utilizing the integral based equation as basic constraint and spatio-temporal local constancy of a motion vector field as additional constraint [10]. Let us consider a square region having $(2 \times L+1) \times(2 \times L+1)$ (pixel $^{2}$ ) as $\delta S$ (Fig.2). Then eq.(2) is rewritten into the following discrete form,

$$
v_{x} I_{x}(x, y, t)+v_{y} I_{y}(x, y, t)+I_{t}(x, y, t)=0,
$$


where,

$$
\begin{aligned}
I_{x}(x, y, t)= & \sum_{m=-L}^{L}\{f(x+L, y+m, t)+f(x+L+1, y+m, t)\} / 2 \\
& -\sum_{m=-L}^{L}\{f(x-L, y+m, t)+f(x-L-1, y+m, t)\} / 2, \\
I_{y}(x, y, t)= & \sum_{l=-L}^{L}\{f(x+l, y+L, t)+f(x+l, y+L+1, t)\} / 2 \\
& -\sum_{l=-L}^{L}\{f(x+l, y-L, t)+f(x+l, y-L-1, t)\} / 2, \\
I_{t}(x, y, t)= & \sum_{l=-L}^{L} \sum_{m=-L}^{L}\{f(x+l, y+m, t+1)-f(x+l, y+m, t-1)\} / 2 .(6)
\end{aligned}
$$

In conversion from the continuous form eq.(2) into discrete form eq.(3), a spatial local constancy of the motion vector field is assumed. Equation (3) is also obtained by a summation of discrete versions of basic constraint equations (1) over $\delta S$. This indicates that the terms: $I_{x}, I_{y}, I_{t}$ with large $L$ are insensitive to random noise in contrast to the partial derivatives: $\partial f / \partial x, \partial f / \partial y, \partial f / \partial t$. Since we have only one constraint equation (3), one more additional constraint equations are required to obtain the set of solutions $v_{x}$ and $v_{y}$. Here we also introduce a temporal constancy of the motion vector field as the additional constraint. From this constancy, we obtain a set of basic constraint equations (3) sharing the same $v_{x}$ and $v_{y}$ along a time coordinate system. Thus we can estimate them by minimizing the following error function,

$$
E=\sum_{n=0}^{N-1}\left\{v_{x} I_{x}(x, y, t+n)+v_{y} I_{y}(x, y, t+n)+I_{t}(x, y, t+n)\right\}^{2},
$$

where $N$ represents frame number utilized for the minimization. Note that $N$ should be greater than 2 for over-deterministic optimization.

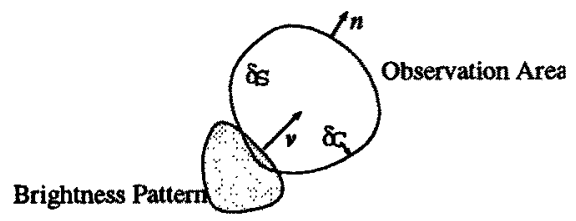

Fig. 1. Graphical representation of the integral based equation (2). Movement $v$ of a brightness pattern causes total brightness change observed in the fixed observation area $\delta S$ surrounded by $\delta C$. The unit length vector $n$ is perpendicular to $\delta C$. 


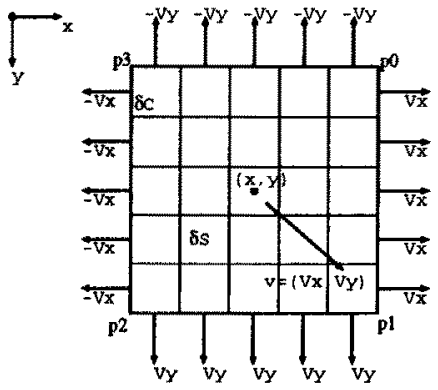

Fig. 2. Graphical representation of the discrete form of the integral based equations (2) and (3). The motion vector components $\left(v_{x}, v_{y}\right)$ are assumed to be constant in the local region $\delta S$. An inner product of $n$ and $v$ becomes $v_{x}$ along the vertical line p0-p1. On the other hand, along the line $\mathrm{p} 2$ - p3, the product becomes $-v_{x}$. In the same way, the product along $\mathrm{p} 1-\mathrm{p} 2$ and the one along $\mathrm{p} 3-\mathrm{p} 0$ become $v_{y}$ and $-v_{y}$, respectively.

\section{Experimental Results}

\subsection{Analysis of Artificially Synthesized Image Sequences}

In this section, we confirm usefulness of the proposed method. For this purpose, we apply the proposed method and a conventional gradient based one to artificially synthesized image sequences and compare them in accuracy. The conventional gradient based method [10] utilizes the basic constraint equation (1) combined with spatio-temporal local constancy of a motion vector field as additional constraint. That is, a motion vector is estimated by minimizing the following error function in the conventional method,

$$
\begin{gathered}
E=\sum_{l=-L}^{L} \sum_{m=-L}^{L} \sum_{n=0}^{N-1}\left\{v_{x} f_{x}(x+l, y+m, t+n)+v_{y} f_{y}(x+l, y+m, t+n)\right. \\
\left.+f_{t}(x+l, y+m, t+n)\right\}^{2},
\end{gathered}
$$

where $f_{x}(x, y, t), f_{y}(x, y, t)$ and $f_{t}(x, y, t)$ are spatial and temporal partial derivatives in discrete form and $(2 \times L+1) \times(2 \times L+1)\left(\right.$ pixel $\left.^{2}\right)$ represents size of a square local region for the minimization. The least squares method is carried out in the minimization. We tentatively call the conventional gradient based method as "conventional Spatio-Temporal Optimization Method (STOM)" and the proposed method as "Proposed Integral Based Method (PIBM)". Both of them assume spatial constancy of a motion vector field within the square region $(2 \times L+1) \times(2 \times L+1)\left(\right.$ pixel $\left.^{2}\right)$ and temporal stationariness of the field during time $N$ (frame).

On the other hand, image sequences are artificially synthesized by,

$$
f(x, y, t)=(1-r) \cdot f_{0}(x, y, t)+r \cdot n()
$$


where $f_{0}(x, y, t)$ represents an image sequence obtained by rotating a real static scene around its center, the function $n()$ generates uniformly distributed random number, $r$ is a ratio between $f_{0}()$ and $n()$. The angular velocity of the rotation is set to 1.0 (degree/frame). For the overall field, a velocity component and a directional one in the rotational motion field distribute from 0 to 1.3 (pixel/frame) and from 0 to $2 \pi$ (radian), respectively. Thus, the image sequence having the rotational motion is useful to the test of accuracy in wide ranges of velocity and direction components. Moreover, varying the ratio $r$ from 0 to 0.5 at intervals 0.1 , we obtain 6 kinds of image sequences. All sequences consists of spatial resolution $150 \times 150\left(\right.$ pixel $\left.^{2}\right)$.

We define a mean error rate of a determined motion vector field to evaluate accuracy of the determined motion vector field by the following equation,

$$
E=\frac{1}{M} \sum_{x} \sum_{y} \frac{\left|v_{t}(x, y)-v_{e}(x, y)\right|}{\left|v_{t}(x, y)\right|} \times 100(\%)
$$

where $M$ represents the number of grid points $(150 \times 150), v_{t}$ is the true motion vector and $v_{e}$ is the evaluated motion vector at each point.

We compare accuracy of the two methods by varying the parameters $N, L$ and $r$. We show their results in Tables 1,2 and 3. From these tables, when we can not utilize so many grid points, say 1 (pixel) $\times 8$ (frame), for determination of a motion vector field, we had better use STOM. Otherwise, PIBM is superior than STOM in accuracy. Furthermore, to evaluate calculation cost we measure their calculation times. As the results, PIBM is about 7 times faster than STOM in calculation time. From these simulation experiments, we understand that PIBM is reliable and it requires less calculation cost than STOM.

\subsection{Analysis of a Real Image Sequence}

Now the proposed method is applied to a real image sequence to test its performance for real scene. The image sequence is generated by a TV camera attached to a passenger seat of a running car in a dark environment in the night. The optical axis of the camera is parallel to the running direction of the car and the camera lens faces toward the front of it. One of the captured image frames is shown in Fig.3. Because of dark environment, its brightness level is very low and thus noise level is relatively high. The two methods: STOM, PIBM are applied to the image sequence. Motion vector fields determined by STOM and by PIBM are shown in Figs.4 (a) and (b), respectively.

From comparison between the two fields, we understand that the field determined by STOM is completely averaged. On the other hand, around the right bottom region in Fig.4(b) we find that motion vectors are in the horizontally right direction which is consistent with the velocity vector field caused by relative motion between the car and the environment. 
Table 1. Dependence of accuracy on the parameter $N$ which represents frame number utilized for the determination of a motion vector field. The parameter $L$ is fixed to be 3 (pixel). The methods STOM and PIBM are applied to a rotational image sequence synthesized with $r=0$.

\begin{tabular}{|c|c|c|c|c|c|}
\hline$N$ & 8 & 16 & 32 & 64 & 128 \\
\hline STOM & 18.1 & 13.9 & 10.3 & 9.15 & 8.27 \\
\hline PIBM & 25.9 & 11.7 & 3.94 & 2.12 & 1.74 \\
\hline
\end{tabular}

Table 2. Dependence of accuracy on the parameter $L$ which represents local size utilized for the determination of a motion vector field. The parameter $N$ is fixed to be 32 (frame). The methods STOM and PIBM are applied to a rotational image sequence synthesized with $r=0$.

\begin{tabular}{|c|c|c|c|c|c|c|}
\hline$L$ & 0 & 1 & 2 & 3 & 4 & 5 \\
\hline \hline STOM & 14.3 & 11.6 & 10.7 & 10.3 & 9.72 & 9.36 \\
\hline PIBM & 25.4 & 9.30 & 5.30 & 3.94 & 3.72 & 3.96 \\
\hline
\end{tabular}

Table 3. Robustness of the two methods STOM and PIBM against to a random noise component. The parameter $r$ represents the ratio between the noise component and rotational image sequence $f_{0}(x, y, t)$. The parameters $N$ and $L$ are fixed to be $N=32$ (frame) and $L=3$ (pixel).

\begin{tabular}{|c|c|c|c|c|c|c|}
\hline$r$ & 0.0 & 0.1 & 0.2 & 0.3 & 0.4 & 0.5 \\
\hline \hline STOM & 10.3 & 15.5 & 28.5 & 42.7 & 51.3 & 55.3 \\
\hline PIBM & 3.94 & 9.27 & 18.6 & 29.6 & 41.2 & 53.0 \\
\hline
\end{tabular}

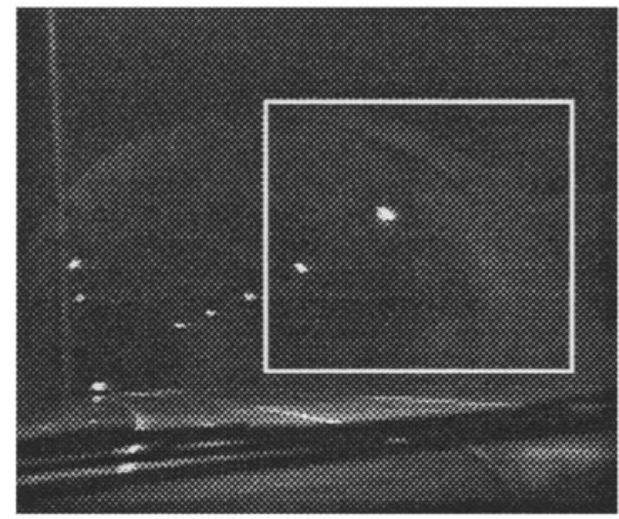

Fig. 3. Real road image sequence acquired from a running car in a dark environment. Sampling frequency is $30 \mathrm{~Hz}$. Brightness is quantized in to 256 levels. Right upper square portion is analyzed. 

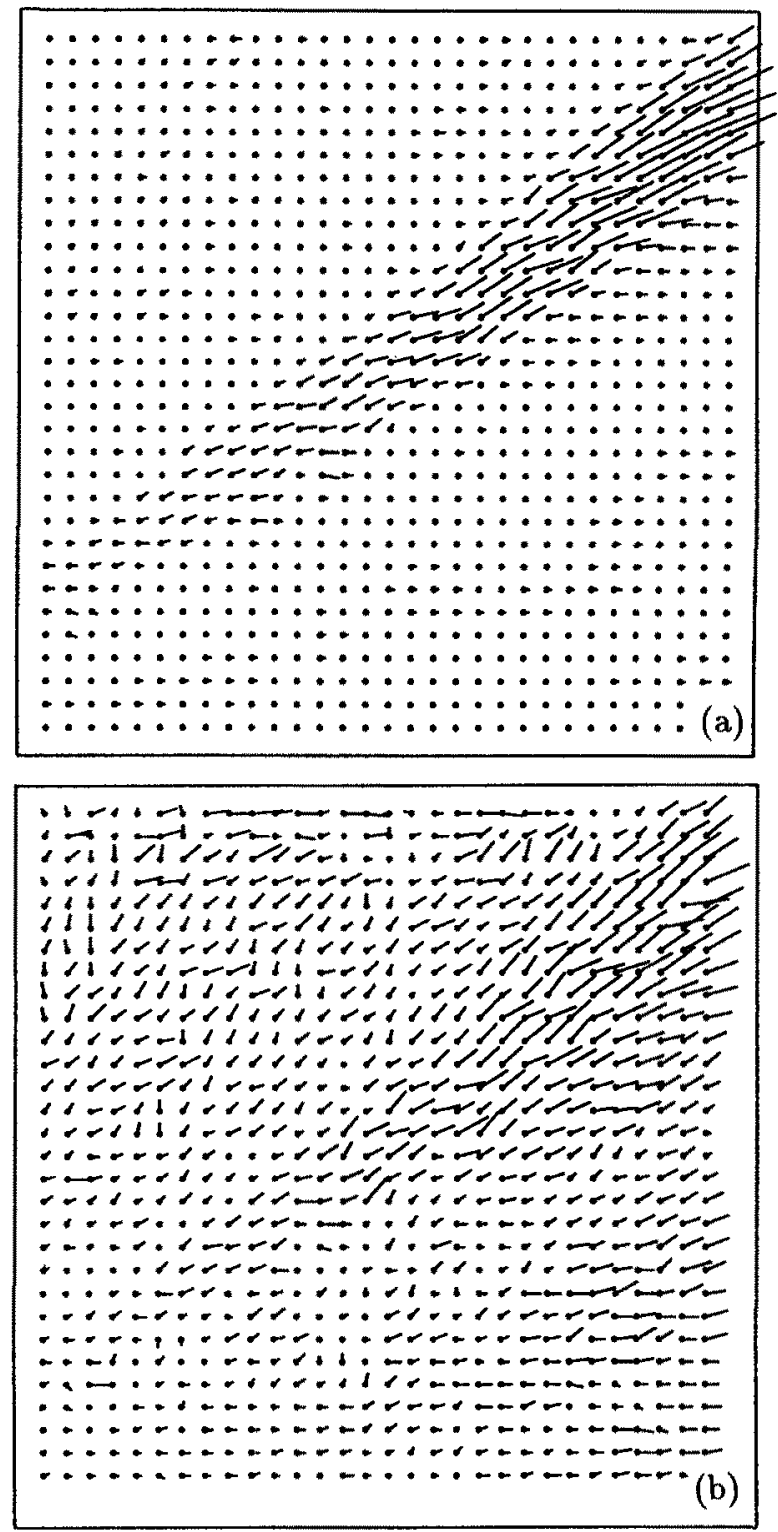

-1[pixel/frame]

Fig. 4. Motion vector fields obtained by (a)STOM and (b)PIBM from the real image sequence(Fig.3). Both methods assume spatial constancy in square portion with $7 \times 7\left(\right.$ pixel $\left.^{2}\right)(L=3)$ and temporal stationariness during 69 (frame) $(N=69)$ corresponding to 2.3 second. 


\section{Conclusion}

In this study, a basic constraint equation in integral form was proposed for the determination of a motion vector field from a noisy image sequence. Moreover, a method employing the proposed basic constraint equation and spatio-temporal local constancy of a motion vector field as an additional constraint was proposed for determination of the field. The proposed method and a conventional gradient based method employing the same additional constraint were applied to artificially synthesized image sequences and a real image one. As the results, the usefulness of the proposed method was confirmed quantitatively for the synthesized image sequence and qualitatively for the real one. In particular, the proposed method was more accurate and it requires less calculation cost.

\section{Acknowledgements}

The author would like to thank Prof. Hidetoshi Miike (Yamaguchi University) for helpful comments and stimulating discussions.

\section{References}

1. S. Maybank: Theory of reconstruction from image motion. Springer-Verlag (1993)

2. P. Anandan: Computing optical flow from two frames of an image sequence. COINS Technical Report 86-16 (1986)

3. B. K. P. Horn and B. G. Schunck: Determining optical flow. Artificial Intelligence 17 (1981) 185-203

4. J. K. Kearney, W. B. Thompson and D. L. Boley: Optical flow estimation: an error analysis of gradient-based methods with local optimization. IEEE Transactions on Pattern Analysis and Machine Intelligence PAMI-9 (1987) 229-244

5. A. Nomura, H. Miike and K. Koga: Field theory approach for determining optical flow. Pattern Recognition Letters 12 (1991) 183-190

6. J. Aisbett: Optical flow with an intensity-weighted smoothing. IEEE Transactions on Pattern Analysis and Machine Intelligence 11 (1989) 512-522

7. A. D. Bimbo, P. Nesi and J. L. C. Sanz: Analysis of optical flow constraints. IEEE Transactions on Image Processing 4 (1995) 460-469

8. N. Mukawa: Estimation of shape, reflection coefficients and illuminant direction from image sequences. Proceedings of the 3rd International Conference on Computer Vision (Osaka, Japan, 1990) 507-512

9. A. Nomura, H. Miike and K. Koga: Determining motion fields under non-uniform illumination. Pattern Recognition Letters 16 (1995) 285-296

10. K. Nakajima, A. Osa, T. Maekawa and H. Miike: Evaluation of body motion by optical flow analysis. Japanese Journal of Applied Physics 36 (1997) 2929-2937 